\title{
Coordination of international multicenter studies: Governance and administrative structure
}

\author{
Shrikant I. Bangdiwala, PhD, (1) C ristiane S. de Paula, MS, (2) \\ Laurie S. Ramiro, PhD, (3) Sergio R. Muñoz, PhD. (4)
}

\section{Bangdiwala SI, De Paula CS, Ramiro LS, Muñoz SR. Coordination of international multicenter studies: Governance and administrative structure. Salud Publica Mex 2003;45:58-66.} The English version of this paper is available too at: http://www.insp.mx/salud/index.html

\begin{abstract}
A bstract
A well-conducted multicenter study needs to assure standardization, unifo rmity of procedures, high data quality, and collaboration across sites. This manuscript describes the organization and dynamics of multicenter studies, focusing on governance and administrative structures among countries of diverse cultures. The organizational structure of a multicenter study is described, and a system for oversight and coordination, along with roles and responsibilities of participants in the multicenter study, are presented.The elements of a governance document are also reviewed, along with guidelines and policies for effective collaboration. The experience of an ongoing multi-country collaboration, the W orld Studies of A buse in the Family Environment (W orld$\mathrm{SAFE})$, illustrates the implementation of these guidelines. It is essential that multicenter studies have an objective coordinating center and that the investigators jointly develop a written governance document to enable collaboration and preserve collegiality among participating investigators. The English version of this paper is available too at: http:// www.insp.mx/salud/index.html
\end{abstract}

Key words: multicenter studies; organization and administration; epidemiologic studies; clinical trials

\author{
Bangdiwala SI, De Paula CS, Ramiro LS, Muñoz SR. \\ Coordinación de estudios multicéntricos internacionales: \\ estructura administrativa y reglamentación. \\ Salud Publica Mex 2003;45:58-66. \\ El texto completo en inglés de este artículo también \\ está disponible en: http://www.insp.mx/salud/index.html
}

\section{Resumen}

La ejecución de estudios multicéntricos requiere el establecimiento de procedimientos uniformes, control de calidad, asegurar estandarización, y la colaboración entre las instituciones participantes. Este trabajo describe la estructura y la dinámica de los estudios multicéntricos internacionales, enfocando los aspectos de administración y reglamentación. Se describe la estructura organizativa de un estudio multicéntrico, así como los roles de los integrantes de un sistema de supervisión y coordinación. Se presentan los elementos de un documento de reglamentación y se describen algunas guías y políticas para una colaboración eficaz. La experiencia del estudio internacional colaborativo W orld Studies of A buse in the Family Environment (W orldSAFE) se utiliza como ejemplo de la aplicación de estas no rmas. U n centro de coordinación estadística, así como un documento de auto-reglamentación son elementos esenciales para establecer y mantener la colaboración de las diferentes instituciones que participan en un estudio multicéntrico. El texto completo en inglés de este artículo también está disponible en: http://www.insp.mx/salud/ index.html

Palabras clave: estudios multicéntricos; organización y administración; estudios epidemiológicos; ensayos clínicos

The work presented here was partially supported by International Clinical Epidemiology N etwork (INCLEN) grant \# CBS 99-001 and by the Chilean "Fondo N acional de Desarrollo Científico y Tecnológico" (FO N DECYT) grant \# 1000232.

(1) University of N orth Carolina, C hapel Hill, N C, USA.

(2) Escola Paulista de Medicina, Universidade Federal de São Paulo, São Paulo, Brazil.

(3) University of the Philippines, Manila, The Philippines.

(4) Universidad de La Frontera, Temuco, Chile.

Received on: September 12,2001 • Accepted on: December 8, 2002

Address requests to: Shrikant I. Bangdiwala, PhD, D epartment of Biostatistics-C SCC , 137 E. Franklin Street, Suite 203,

Chapel Hill, N C 27514-4145 USA

E-mail: kant@ unc.edu 
E very research study requires a sound design, careful planning, good management, and proper analysis. In some cases, a study is conducted in multiple sites due to sample size considerations, the desire to explore differences among sites, or for enabling generalization of results across sites. It should be noted that there is a distinction between multi-site studies and multicenter studies. In both types of studies, multiple institutions perform the same procedures as others. However, in multi-site studies, the investigators at the sites do not participate as co-investigators of the study; they are merely carrying out the study (e.g. recruiting subjects, treating subjects, and/or following subjects) and thus can be viewed as contractors. On the other hand, in a multicenter study, the investigators at the sites are involved as co-investigators in the planning of the study protocol and procedures, are scientifically responsible for the study results, and participate in manuscripts and other dissemination activities.

This paper presents the organizational structure for a multicenter study that is international, discussing the roles and functioning of the participating centers and committees. The elements of a governance document and of publication guidelines are also presented. The World Studies of Abuse in the Family Environment ${ }^{1}$ are used as an example on the feasibility of implementing the ideas of a coordinating center, oversight for a study, and governance documents internationally.

\section{Organizational structure of multicenter studies}

In a multicenter study, it is necessary that the activities be shared among the principal investigators from each of the participating centers. Each must feel scientifically responsible and accountable for the integrity of the study. ${ }^{2}$ Oversight and policy making in such studies is provided by the Steering Committee (SC), composed of the principal investigators of each participating center. Depending on the complexity of the study, there may be other central agencies (e.g. central laboratories, event adjudication committees) or external committees (e.g. data and safety monitoring board, technical advisory committee, sponsor).

If a study is conducted in multiple sites, a major effort is necessary for assuring standardization and uniformity across sites. In such studies, it is necessary to have an independent group, not one of the sites, to be held responsible for assuring and monitoring the proper conduct of the study. ${ }^{3}$ This institution is called the Statistical Coordinating Center (CC). The roles of the CC are two-fold: overall study coordination and management, and statistical data management coordination. Though not commonly done, in some cases, one institution may perform the administrative tasks of the CC while another institution is in charge of the statistical and data handling tasks.

The typical organizational structure can be explained by grouping the roles into three levels: oversight level (Sponsor and Steering Committee), coordination level (Coordinating Center and other Centralized Agencies) and conduct level (Site specific) see figure 1 .

For example, the World Studies of Abuse in the Family Environment (WorldSAFE) is a multi-center observational study whose primary objective is to investigate child and spousal abuse that occurs within families in different cultures across a variety of countries. To date, five countries are involved: Brazil, Chile, Egypt, India and Philippines, and the study is still recruiting new sites. Figure 2 describes the organizational structure of WorldSAFE. There are a total of 11 sites participating, with one site per country except for India, which received additional country-specific funding and thus was able to maintain the seven separate sites. Because of the multiple sites within India, there is a separate coordinating center for Indian sites.

\section{Oversight level}

All studies, whether at a single site or multi-site, require overall guidance and a policy-making group. Single site studies have one person typically responsible

0 versight level
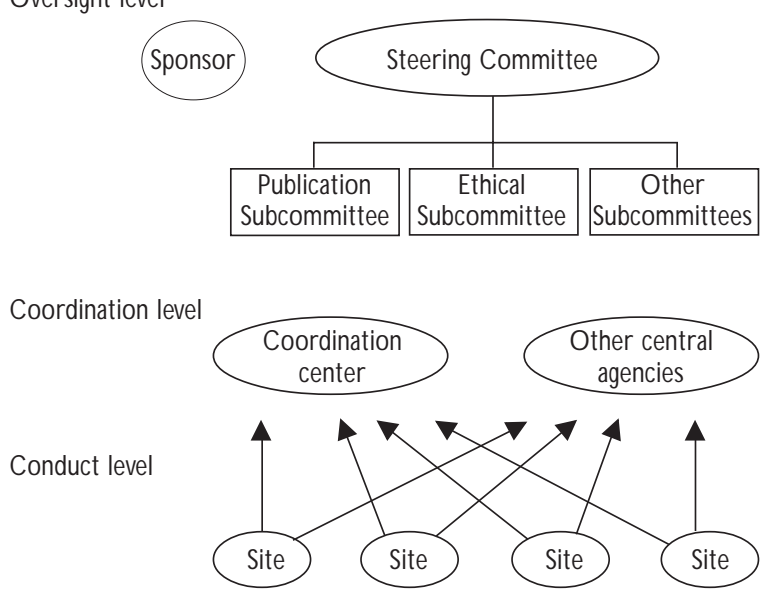

Figure 1. Structure of a typical multicenter study 


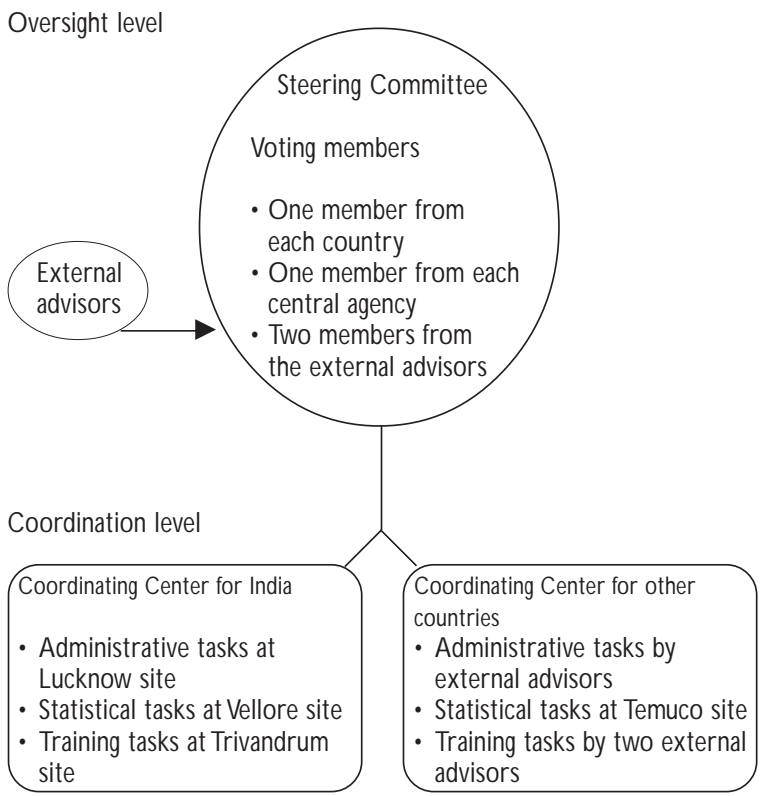

Conduct level

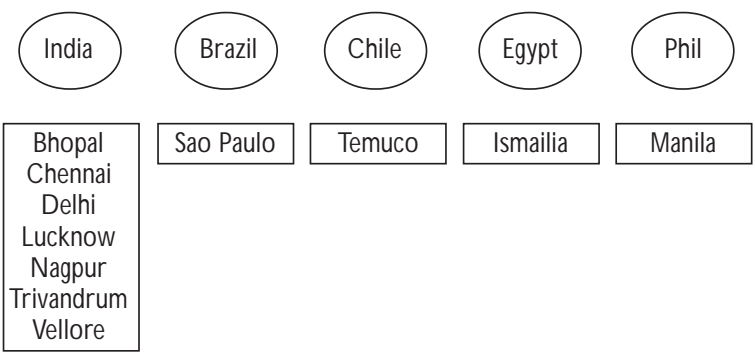

Figure 2. WorldSAFe Organizational Structure

for this activity, the study principal investigator (PI). However, multicenter studies involving multiple PIs must unite the various investigators into a policy issuing/governing group. In addition to internal oversight, external oversight from advisory boards and the study sponsor are often part of the study.

The study steering committee (SC) is responsible for providing the overall guidance for the study, setting policy, and for the dissemination of study results. Since this group is scientifically responsible for all study decisions, the members of the SC should include representation from each study institution, from sites as well as from centralized agencies. Although an institution may have two or more investigators on the committee, typically the one voting member per institution is the principal investigator.

It is often difficult for the SC to meet frequently, an issue that is more challenging for studies done in- ternationally. Depending on the size of the committee, and of the financial status of the study, the SC may elect to hold conference calls, or communicate frequently on decision making issues via electronic mail, a secure web server, closed chat rooms, or other rapid systems such as faxes or courier services. However, there is always a need for face-to-face communication and discussion of issues, and it may be necessary for a subset of the SC to be designated as an Executive Subcommittee, a much smaller group of usually 3-5 individuals for more frequent interaction. Conference calls, though costly, are cheaper than in-person meetings and especially useful for multi-country studies.

The SC, again depending on its size, often also surrounds itself with various subcommittees to develop and monitor study activities (e.g. recruitment, measures, publications, data handling, etc.). These subcommittees function as advisors to the SC, i.e. they meet more frequently to study issues in depth, elaborate proposed policies, present them to the full SC for ultimate decision taking, and monitor the implementation of the policies.

For example, the WorldSAFE Steering Committee is composed of the principal investigators of the countries, of the coordinating centers, and of two out of the four external consultants to the study. The investigators in India have also a country-specific steering committee. Neither is large enough to have subcommittees.

The study sponsor also provides some oversight, but is responsible primarily for financial support. The sponsor can be a government agency, a private industry organization, a volunteer non-profit organization, a charitable foundation, or a third-party payer. The sponsor may be invited to participate in providing oversight to the study investigators, may be solely relegated to being a financial provider, or may take an active role in the oversight of the study. The different levels of involvement of the sponsor depend on the desires of the study investigators or of the sponsor, and of the acceptability by the scientific community of their participation.

In many studies, an independent group of advisors external to the study institutions is formed to provide oversight and guidance. In addition, in clinical trials where ethical considerations from conducting an experiment dictate the needs, a data and safety monitoring board (DSMB) is often mandatory. The DSMB members include investigators, biostatisticians, and ethicists that are not from any of the study participating institutions and thus have no potential conflict of interest with study results. The DSMB is responsible for external oversight, patient safety (adverse events), study integrity, and interim analyses (for the possibili- 
ty of early termination). It should be noted that the roles of the DSMB differ from each participating institution's ethics board or IRB (Institutional Review Board), who approve the ethical conduct of the study at their institution, but typically do not monitor interim analyses given their potential institutional conflict of interest.

\section{Coordination level}

The unique aspect of a multi-site study is the existence and the essential need for coordination and centralization of activities. For example, protocol development, development of study materials, training, communication, laboratory determinations, data processing and management, report generation, statistical analysis, and manuscript development, are all activities that are common to any study, whether single-site or multi-site. However, in a multi-site study, these activities are often centralized because of the need to standardize them across all sites, which often also has the further benefit of resulting in a gain of cost efficiency.

However, when the activities are centralized, the central group 'controls' the data and hence the study; and must take steps to assure other participating institutions that it is managing it adequately. This assurance is provided by developing adequate systems for staff training and quality assurance, collecting, entering, managing and analyzing the data. These activities are done by the statistical coordinating center (CC) (table I). In addition, it is imperative that communication among all participating institutions be clear, continuous, and well documented, a major task of the coordinating center. Finally, but maybe the most important task of the centralized institutions is that of assuring the collaboration of all study participating institutions in having their data aggregated and shared with others. It is thus necessary that written rules and regulations be elaborated to give equal and fair access to all participating investigators -this is where governance documents are relevant (Table II).

One important responsibility of the CC is the administrative coordination of the study. It is the main center for communication between all participating sites, ensuring that sites receive notification of changes to the protocol or forms, clarifications of the manual of operations, patient safety updates, and other critical information. The CC is also responsible for supporting study functions: meeting regulatory requirements, producing progress reports for the study investigators and sponsor, facilitating communications within the study, and managing data security systems. ${ }^{4}$ They also coordinate and facilitate meetings of the investigators' committees and of external committees.

Table I

\section{Functions of a Coordinating Center}

General areas

Statistical and content methodological support (quantitative and qualitative data)
Specific functions

Planning phase

- Define study objectives

- Develop methods and procedures

- Estimate sample size requirements

- Identify the data to be collected and the collection methodology

- Define the important outcome measures

- Edit sections of the protocol and study manuals

Conduct phase

- Prepare data analyses and monitoring reports

- Assure the integrity of data

- Establish and run the quality assurance procedure

Analysis phase

- Conduct proper statistical analyses of aggregate data

- Assure adequate content and methodological input in analyses

- Assure that valid reports and manuscripts are produced

\begin{tabular}{ll}
\hline Systems coordination, & Establish systems for data collection and pro- \\
communications & cessing \\
& Establish lines of communication and docu- \\
& mentation \\
\hline
\end{tabular}

Computer system support - Develop data management systems

and programming - Develop systems for tracking patients and forms

- Design periodic interim reports

- Develop and execute programs for statistical data analysis

- Monitor operations and budget

Study material creation - Design/preparation and distribution of study and management materials (final Protocol, forms and documentation)

- Develop training materials, operation manuals for resource centers, for management of drugs and specimens

- Manage data collection and centralization

- Manage protocol amendments and revisions

Training and performance - Conduct quality assurance and performance monitoring monitoring

- Evaluate the effectiveness of training

Data operation - Coordinate drug distribution

- Coordinate activities of centralized agencies and clinical sites

- Manage data collection, transmission, review, entry, and editing

- Handle quality control problems

- Maintain aggregate database

Administrative support - Responsible for communications, routine reporting

- O rganize study committee meetings, prepare minutes

- Decision on microcomputers and software to be used by all participating institutions

Documentation and archival - Appropriate processing and storage of study records

- Backup copies of the database and system with documentation 
Table II

\section{Contents of a typical governance document}

\begin{tabular}{l} 
Introduction \\
- D escription of the study, all participating institutions and individuals \\
- Mission statement of the governance document \\
\hline Study organization \\
- $\quad$ Roles and responsibilities of the participating institutions and indi- \\
viduals \\
- D efinitions of principal investigators and co-investigators \\
- $\quad$ Specification of the administrative structure for self-governance \\
Decision making policies \\
- D esign and protocol amendments \\
- Voting rights and majority rules \\
\hline Data access and sharing \\
- Core (pooled) data, site-specific data
\end{tabular}

Publications and co-authorship

The essential CC staff member for these tasks is the project coordinator (PC), the focal person for communication and study management. This individual is responsible for resolving questions and issues raised by the sites, helping the site staff with data transfer, and any other aspect of supporting the operations at the sites. Often called the data coordinator, data manager or data specialist, (s)he is responsible for quality control of the data; for generating edit queries and data requests; and for maintaining all study files. The role of coordinating and maintaining communication is quite challenging even if a multicenter study is conducted in a single country, but it is substantially more complex in multi-national studies (table III). For example, sending faxes to countries where the same phone is used for voice as well as for fax during local office hours, cannot be automatic, and require that the sender know the local language to communicate that a fax is being sent. Aside from the usual difficulties with communication infrastructure that one should expect to encounter when some of the countries are in lesser stages of development, challenges arise when cultural differences are encountered. Thus, in cultures that find it difficult to say 'no,' proposed changes to the protocol by one group may not be objected to in any verbal or written communication, but may not be carried out later by that site. In addition, language differences complicate interactions not only in communications but also for translating and back translating study materials.

The second major responsibility of the CC is ensuring the quality of the data in all its aspects: design of data collection forms, field work procedures, data entry management systems, quality assurance procedures, data processing and editing, and analysis and interpretation of data. The PI of the CC assumes ulti-

Table III

\section{EXAMPLE OF COMMUNICATION SYSTEMS NECESSARY IN A MULTICENTER STUDY}

\begin{tabular}{|c|c|c|}
\hline Other Centers (OCs) & From OCs to Coordinating Center (CC) & From CC to OCs \\
\hline $\begin{array}{l}\text { Sponsor } \\
\text { Study } \\
\text { Leadership }\end{array}$ & $\begin{array}{l}\text { - O peration funds } \\
\text { - Projections regarding future funding } \\
\text { - O verall scientific guidance } \\
\text { - Priorities }\end{array}$ & $\begin{array}{l}\text { - Reports of resources utilization level } \\
\text { - Reports on study progress and performance of all centers } \\
\text { - Statistical and clinical trial principles } \\
\text { - Reports on study progress and database quality } \\
\text { - Suggestions of forms and protocol refinement } \\
\text { - Suggestion for policy change } \\
\text { - Reports of resource availability } \\
\text { - Progress of manuscripts }\end{array}$ \\
\hline $\begin{array}{l}\text { Centralized } \\
\text { agencies }\end{array}$ & $\begin{array}{l}\text { - Data } \\
\text { - Identification of system problems relative, } \\
\text { to data quality or timeliness }\end{array}$ & $\begin{array}{l}\text { - Training } \\
\text { - Study materials supplies by CC } \\
\text { - Reports, tracking submission to CC } \\
\text { - Request for resolution of data inconsistencies } \\
\text { - Reports of performance and progress } \\
\text { - N otification of data quality/timeliness problems }\end{array}$ \\
\hline Sites & $\begin{array}{l}\text { - Data } \\
\text { - Identification of system problems relative } \\
\text { to data quality or timeliness }\end{array}$ & $\begin{array}{l}\text { - Training } \\
\text { - Study materials supplies by CC } \\
\text { - Reports of enrollments } \\
\text { - Reports tracking specimen and data flow from field centers to resource center } \\
\text { - Request for resolution of data inconsistencies } \\
\text { - Reports of performance } \\
\text { - Announcements of cutoff dates for data monitoring committee analyses } \\
\text { - N otification of data quality/timeliness problems }\end{array}$ \\
\hline
\end{tabular}


mate responsibility for the quality of the data; the determination of adequate sample size; for defining the statistical methodology; for data analyses of the stu$\mathrm{dy}$; and is involved in monitoring the progress of the study. The PI is a member of the study steering committee (SC).

Computer programming staff at the $\mathrm{CC}$ are responsible for designing and creating the data entry management system. Often, the system consists of a core common database for all sites, with additional site-specific data modules. The core common database is a frequent situation in multi-center studies since many of the site investigators may not choose to collect some part of the information that others collect; also, some questions may not be considered culturally appropriate in some settings. Typically the data entry management systems are in a common language, while site-specific instruments are in the local language, which may pose a problem for site data entry operators. Programmers are also responsible for the ongoing maintenance, including installation of software upgrades, for ensuring database integrity and security and for maintaining an adequate backup system. It is a challenging activity when, because of financial limitations, the various sites have different computer set-ups, with different versions of common software, different software, different operating systems or even different platforms. It is also more difficult to protect such a network of computers from viruses and to provide on-site maintenance, so that it is often more practical to have local providers maintain the systems.

Statistical staff at the CC are responsible for analyzing the data, including preparation of periodic reports for monitoring progress and quality assurance, as well as descriptive and inferential analyses of study results. When a particular study design combines the depth of qualitative data and breath of quantitative research, the CC should expand its staff capabilities with the addition of appropriate social scientists to the team. These co-investigators are responsible for the qualitative study design (sample selection, methods and techniques of data collection, instrumentation), its quality and analyses. The nature of the analysis methodology may require that each study site do the interpretation of their results based on themes common across all sites but in accordance with their own cultural distinctions.

Periodic internal and external reports of the CC monitoring study progress must also detail data collection irregularities, timeliness and completion of forms, data entry errors, inconsistencies, and other aspects of quality assurance. An integral component of quality assurance is the training of the study staff.
The training can be done by workshops, via video or internet methods, and/or with written material. The training is important to ensure that staff understand the protocol and the requirements of the study. Once the study is underway, continuing training efforts may be necessary, especially if there is staff turnover. Internationally, this is difficult to achieve from a budget standpoint, and it is often the case that the site PI or data coordinator is trained so that that individual can provide training for new staff ('train the trainer approach').

Aside from centralized coordination of data in a multicenter study, laboratory determinations benefit from being centralized since standardization of reagents and of laboratory procedures is easier to implement. On the other hand, difficulties lie in the need to establish reliable (and often rapid) means of transporting the samples to the central laboratory. Cost is an important issue, but Customs and in-transit country restrictions on transport of human specimens need to be fully accounted for.

As mentioned above, WorldSAFE has two coordinating centers, one for the India sites and one for other countries in Temuco, Chile. The coordinating center (CC) tasks for the India sites are actually shared among three of the seven sites, with Lucknow serving as the $\mathrm{CC}$ for administrative tasks, the training activities being coordinated by the Trivandrum site, and the statistical CC tasks performed at the Vellore site.

The CC tasks for WorldSAFE sites not in India are also shared among different organisms, due to financial considerations. Thus the statistical tasks are located at the Temuco site, the administrative tasks are performed by the team of four external advisors, and the training tasks are provided by the external advisors. The training for all of the WorldSAFE sites including India was provided to each country by the same team of external advisors, and the within-country training was provided by those trained if there were more than one site (only in India).

Given that there are two coordinating centers performing statistical tasks in WorldSAFE, an additional collaboration and coordination was necessary. Thus, the data entry software for the common data form was first developed by the Vellore CC for all India sites, and it served as the basis for the one developed by the Temuco CC for all other participating countries. Common data cleaning and management procedures were developed at both CCs in co-operation with one of the external advisors. Data files for all sites are available at both CCs, while each of the corresponding CC is responsible for the updates to their corresponding sites' databases. Analyses can be performed at either CC, 
subject to the process established by the Steering Committee.

The lack of funding for the CC to undertake the administrative tasks for WorldSAFE implied that continuing education, protocol clarifications and other support of sites was not continuous, but sporadic; it took place at the irregularly-spaced meetings of investigators. Specific questions were addressed by e-mail from sites to one of the advisors or to the CC in Temuco and other priorities hindered rapid communication. Ad-hoc subcommittees of the SC adopted some tasks until adequate funding for the $\mathrm{CC}$ is obtained.

The two CCs of WorldSAFE, one in Vellore specifically for India and the other in Temuco, made the communications within a region/country more efficient, but more difficult as a whole. Given that the level of funding was not comparable between the two CCs, the level of support each was able to provide to the sites for which they were responsible, was not uniform. Communication and sharing of data between the two CCs was thus more cumbersome. E-mail and internet-based communication have kept costs down.

\section{Conduct level}

The sites are responsible for direct interaction with the study participants, being responsible for identifying and screening potential study participants, obtaining informed consent, enrolling eligible study participants, collecting data (interviews), scheduling participant visits, maximizing patients' compliance, and minimizing loss to follow up. Sites are responsible for routine communications with the CC in order to resolve problems with data collection and submission; and for data transmissions. Finally, sites are responsible for complying with country regulations and ethical guidelines. These issues include obtaining appropriate ethical review, and complying with informed consent procedures. The site PI is scientifically responsible and accountable for the conduct of the study and all site activities, and is a member of the steering committee.

The site data coordinator is responsible for collection, quality, and management of data from the site. This person has to schedule the interviews, supervise the conduct of interviews, supervise the interviewers and process the questionnaires/interviews. The data coordinator is the contact person for the coordinating center's project coordinator (PC). They interact on a very frequent basis, to keep up with the activities of the study. At a minimum, week-to-week contact is necessary, and this is often done via e-mail communication internationally. If a local site handles their sitespecific analyses, or if the study permits local sites to analyze cross-site data, ${ }^{1}$ sites may need to have statistical and computer programming staff. The site statistician is usually responsible for site-specific analyses only.

\section{Governance}

One of the special needs for any study at multiple institutions is the additional complexity of getting a large number of investigators to collaborate and to follow a common protocol. Single site studies may also have multiple investigators, but the single principal investigator usually is able to exercise authority in obtaining the necessary standardization. This is similar to a multi-site study, since the site investigators do not have a role as participating investigators in protocol design, nor in analyses and publications. However, in multicenter studies, the complexity arises because of multiple principal investigators at the different participating institutions, as well as other affiliated investigators. The main purpose of a governance document is thus to provide written rules and regulations by which the investigators will self-govern, with the objective of furthering the common good.

The compendium of guidelines for co-operation among the participating institutions of a multi-center study is collectively called the Governance Document (GD). This may be a simple and short document, but often it is laborious in detail and is constantly in flux. The GD should have the approval of all sites' principal investigators. The elements of a governance document are listed in table II. The first two sections are primarily to specify the committee structure for the study, and to clarify the roles and responsibilities of all involved. The next section delineates how the institutions will interact in decision making. The two last sections are usually the most controversial, since they involve sharing the data (issues of access) and sharing the academic rewards of the research (issue of publications and co-authorship). There is no perfect model, and each study will have to struggle with setting their own guidelines, but some broad issues to consider are mentioned below.

\section{Data access}

Access to data in a multicenter study is more complex than data from a single-site study. This is so because there are data that are site-specific and data that are 'common.' The common data are pooled into an 'aggregate database' at the CC. Typical issues that arise include: (i) who has access to the aggregated data; (ii) does site A have access to Site B's site-specific data; 
(iii) if a site investigator leaves the study, do they retain access? Does the replacement gain access; and (iv) when do other non-study investigators gain access to the data? Who decides?

These decisions are complex since they involve many decision makers. Investigators who are not part of the study usually must submit a written proposal to gain access. Decisions and policies are typically handled by the Publications Subcommittee, but the ultimate policy setting authority resides with the Steering Committee.

\section{Publication policies}

The Publications Subcommittee has the additional difficult task of setting guidelines for collaborating on publications, other dissemination activities such as scientific and non-scientific presentations, and co-authorship. Publication policies are established by the Publications Subcommittee and approved by the Steering Committee. These include policies for all presentations, abstracts, scientific publications, seminars, press releases or other dissemination activities. Typically, local sites are allowed to publish analyses of their site-specific and their common data; it is also collegial courtesy to inform other study investigators of these activities. Publications that use pooled data are more problematic. All such publications must go through approval and follow the process established by the Publications Subcommittee. Typical issues that arise include: (i) investigator $X$ wants to publish the same idea as investigator Y; (ii) investigator W does not want his/her site's data used in a manuscript proposed by investigator $\mathrm{Z}$; and (iii) investigator $\mathrm{S}$ is afraid that investigator $T$ will publish results from their site before (s)he has a chance to publish results from his/her own site.

The above legitimate concerns from investigators participating in a collaborative effort are what the policies set forth in the publication guidelines should address. The approach used in WorldSAFE is the democratic approach of allowing all investigators to provide ideas for analyses of the common data, and allow all to have the possibility of working on a manuscript proposed by others (non-facility in the English language may be an impediment in some multi-national studies). Some of these concepts may not apply well in some cultures, and fairness to all participants may need to be sensitive to this aspect.

\section{Authorship guidelines}

Finally, the most difficult issue is establishing rules for co-authorship. Some investigators may assume that participating in a multi-center study guarantees that they will be co-authors of all publications of the common data. This may not be manageable in large studies, and unless decisions are made collectively early on in the study, this may lead to unconstructive situations. Many scientific journals require that only those who had substantive contributions in the writing of the manuscript can be listed as co-authors. The decision of 'substantive contribution' needs to be made; by whom and under what criteria, must be specified.

Guidelines for co-authorship have also been adopted by many academic institutions, and are based on the premise that authorship is not conferred, but earned, and that all study participants be given equal and fair opportunities to earn the co-authorship. Earning co-authorship requires an 'intellectual' contribution to a manuscript as well as actual contribution to the writing of the paper. For example, solely contributing subjects does not merit co-authorship, nor does simply performing statistical calculations.

All study investigators and personnel must have the opportunity to participate in manuscripts. The participation procedure is determined by the Publications Subcommittee. A common approach, also used in WorldSAFE, is to form manuscript specific 'working groups' among all those interested in a particular topic. It is typical that in large collaborative efforts that some working groups may be quite large; in practice, a 'facilitator' keeps the group moving towards development of the manuscript, and typically only a few members of the group choose to actively participate. Authors for a particular manuscript are then the actively participating members of the manuscript working group. Also, authorship order is based upon their relative contribution to the manuscript. Definitions of 'actively,' 'substantive,' and 'intellectual' contributions are usually specified by the Publications Subcommittee, and involve more than revising drafts for grammar and typographical errors, for example. Publications guidelines include also acknowledgements guidelines for sites, the coordinating center, and other study participants.

When data are collected at many sites and in different countries, it is typically expected that all manuscripts would have representation at least from each country, but the method suggested above does not guarantee this. The above method implies that each investigator needs to earn their co-authorship in every manuscript. Investigators that are culturally 'passive collaborators' may be affected by the above method. Also, in a collaborative effort, a particular manuscript may not include data from a particular site, but the investigators from that site are permitted to join the working group if interested. It may also occur that data 
from a site are used in a particular manuscript and no one from that site is a co-author; in such a case the site is typically acknowledged only. If a site refuses to have their data shared, the collaboration fails.

While the suggested publication policies above may sound harsh and some investigators may feel they are 'not necessary, since we are all collegial friends,' they are meant to ensure that all study members have the same opportunity to participate in presentations and publications, fairly.

When the common data reside at the CC in another country, Investigator $X$ in a different country may not feel that he or she has the same access as an investigator at the $\mathrm{CC}$ and a copy of the analyses files may be sent to each site for exploratory analyses. However, it is in the best interest of a multi-center study that final statistical analyses for manuscripts be centralized at the CC, to assure use of the latest updated and corrected files, and the use of standard data procedures across the study. However, distributed data analyses may lead to more publications if the centralized resources are not adequate. ${ }^{5}$ Internationally, access to data is a challenge for rapid interaction, discussion, conducting statistical analyses, reviewing results, and so forth. Recent advances in communication technology have made this more possible. For example, it is now possible to use the internet and with password protection, have specific access to servers and web pages, to communicate, download data, post analyses results, and drafts of manuscripts for participants anywhere to be able to interact in developing publications.

WorldSAFE's experience with governance has been mixed. Antagonistic issues have not arisen to test the governance document. Collaborative publications have not been hindered by conflicts, but more from lack of time and other priorities for investigators with limited funding support.

\section{Discussion}

The issues presented in this manuscript are ones that must be considered in the planning of health studies internationally. The issues raised must be adapted to the specific circumstances of any particular project. Cultural sensitivities are important considerations, and these especially impact governance methods adopted by the project. From a rigor standpoint, one must be flexible but still attend to the methodological considerations for proper planning and conduct of international collaborative efforts in health research. The experience of the WorldSAFE study leads to a few recommendations. Effective international communication and coordination is best done with a single coordinating center that assumes scientific responsibility for the overall study data. Regional coordinating centers or country-specific coordinating centers can provide more immediate support to local sites, but they in turn should be coordinated by one adequately-funded center.

\section{References}

1. International Clinical Epidemiology N etwork 2000; W orldSAFE and IndiaSAFE. Studying the prevalence of family violence, Philadelphia (PA): INCLEN Monograph Series on Critical International Health Issues No.9.

2. Mowery RL, W illiams OD. Aspects of clinic monitoring in large-scale multiclinic trials. Clin Pharmacol Ther 1979;25:717-719.

3. Bangdiwala SI, Paton CC, Jackson M R. C linic monitoring in collaborative clinical studies. En:O kuno T, ed. Biometry. C linical trials and related topics. Amsterdam: Excerpta Medica, 1988:37-46.

4. Blumenstein BA, James KE, Lind BK, Mitchell HE. Functions and organization of coordinating centers for multicenter studies. Controlled Clin Trials 1995;16:4s-29s.

5. Perkins LL, Cutter GR, W agenknecht LE, Savage PJ, D yer AR, Birch R. D istributed data analysis in a multicenter study:The CARD IA Study. Controlled Clin Trials 1992;13:80-90. 